Prev Med. 2015 October ; 79: 5-14. doi:10.1016/j.ypmed.2015.06.002.

\title{
Firearm injuries in the United States
}

\author{
Katherine A. Fowler ${ }^{a,}{ }^{*}$, Linda L. Dahlberg ${ }^{a}$, Tadesse Haileyesus ${ }^{b}$, and Joseph L. Annest ${ }^{b}$ \\ ${ }^{a}$ Division of Violence Prevention, National Center for Injury Prevention, Centers for Disease \\ Control and Prevention, Atlanta, GA, United States \\ bDivision of Analysis, Research, and Practice Integration, National Center for Injury Prevention, \\ Centers for Disease Control and Prevention, Atlanta, GA, United States
}

\begin{abstract}
Objective-This paper examines the epidemiology of fatal and nonfatal firearm violence in the United States. Trends over two decades in homicide, assault, self-directed and unintentional firearm injuries are described along with current demographic characteristics of victimization and health impact.
\end{abstract}

Method-Fatal firearm injury data were obtained from the National Vital Statistics System (NVSS). Nonfatal firearm injury data were obtained from the National Electronic Injury Surveillance System (NEISS). Trends were tested using Joinpoint regression analyses. CDC Cost of Injury modules were used to estimate costs associated with firearm deaths and injuries.

Results-More than 32,000 persons die and over 67,000 persons are injured by firearms each year. Case fatality rates are highest for self-harm related firearm injuries, followed by assaultrelated injuries. Males, racial/ethnic minority populations, and young Americans (with the exception of firearm suicide) are disproportionately affected. The severity of such injuries is distributed relatively evenly across outcomes from outpatient treatment to hospitalization to death. Firearm injuries result in over $\$ 48$ billion in medical and work loss costs annually, particularly fatal firearm injuries. From 1993 to 1999, rates of firearm violence declined significantly. Declines were seen in both fatal and nonfatal firearm violence and across all types of intent. While unintentional firearm deaths continued to decline from 2000 to 2012, firearm suicides increased and nonfatal firearm assaults increased to their highest level since 1995.

Conclusion-Firearm injuries are an important public health problem in the United States, contributing substantially each year to premature death, illness, and disability. Understanding the nature and impact of the problem is only a first step toward preventing firearm violence. A science-driven approach to understand risk and protective factors and identify effective solutions is key to achieving measurable reductions in firearm violence.

\section{Keywords}

Violence; Firearms; Epidemiology

*Corresponding author at: Division of Violence Prevention, Centers for Disease Control and Prevention, 4770 Buford Hwy NE, MS F-64, Atlanta, GA 30341, United States. kafowler@cdc.gov (K.A. Fowler).

Conflict of interest

The authors declare that there are no conflicts of interests. 


\section{Introduction}

The tragedy in Newtown, Connecticut on December 14, 2012 cast a spotlight on firearm violence in the United States. Twenty-seven people, mostly schoolchildren and their teachers, lost their lives that day. It was the deadliest school shooting in an elementary or high-school in U.S. history. In an average week, 645 people lose their lives to firearm violence and 1565 more are treated in an emergency department for a firearm-related injury (Centers for Disease Control and Prevention, National Center for Injury Prevention and Control, 2005). Most of these events do not make headlines, yet reflect part of the human toll of firearm violence in the United States.

The nature and frequency of firearm violence, combined with its substantial impact on the health and safety of Americans, make it an important public health problem. Many Americans are non-fatally injured or die in acts involving a firearm each year in the United States. These include acts of interpersonal violence, self-directed violence, legal intervention (i.e., injuries inflicted by law enforcement during the course of duty), unintentional injuries involving a firearm, and acts where the intent cannot be determined. Firearm-related injuries are highly lethal and account for $7.1 \%$ of premature death or years of potential life lost before the age of 65 (Centers for Disease Control and Prevention, National Center for Injury Prevention and Control, 2005). Firearm homicide is the second leading cause of injury death among youth 10-24 years of age. Firearm suicide, on the other hand, is the third leading cause of injury death for persons aged 35 years and older, after drug overdoses and motor vehicle crashes. Overall, firearm injuries are among the 5 leading causes of death for people ages 1-64 in the United States.

Firearm violence is preventable. The first step in preventing it is to understand the nature and extent of the problem - what it is, whom it affects, where it occurs, how patterns have changed over time and the factors contributing to these changes. An examination of the factors contributing to firearm violence and changes over time is covered elsewhere in this special issue. Here we provide an overview of fatal and nonfatal firearm violence in the United States-examining patterns of interpersonal, self-directed and unintentional firearm injuries and deaths, including the demographic characteristics of victimization, trends over time, and health impact.

\section{Methods}

A firearm-related injury is defined as a gunshot wound or penetrating injury from a weapon that uses a powder charge to fire a projectile. This definition includes gunshot injuries sustained from handguns, rifles, and shotguns but excludes gunshot wounds from airpowered, gas-powered, BB and pellet guns, as well as non-penetrating injuries associated with firearms (e.g., "pistol whipping").

Fatal firearm injuries were derived from death certificate data from the National Vital Statistics System (NVSS), operated by CDC's National Center for Health Statistics, and were obtained via CDC's Web-based Injury Statistics Query and Reporting System (WISQARS) (Centers for Disease Control and Prevention, National Center for Injury 
Prevention and Control, 2005). Firearm deaths were examined by known intent ${ }^{1}$ (homicide, suicide, unintentional), age, race/ethnicity, and geographic region.

Data on nonfatal firearm injuries from 1993 through 2012 were from the National Electronic Injury Surveillance System (NEISS), which is operated by the U.S. Consumer Product Safety Commission (CPSC) (U.S. Consumer Products Safety Commission, 2014). Data were obtained through an interagency agreement between CDC and CPSC for an ongoing special study called the NEISS Nonfatal Firearm Injury Surveillance Study. NEISS is a stratified probability sample of 99 U.S. hospitals that have an emergency department (ED) and a minimum of 6 beds. Nonfatal injury estimates have been adjusted to account for hospital nonresponse and changes in the number of US hospital EDs over time. NEISS classifies injury intent using standard definitions for the following categories: assault, selfharm, unintentional, and legal intervention. Information on nonfatal injury by racial/ethnic group is not presented here due to large amounts of missing race/ethnicity data. ${ }^{2}$ Status when released from the ED (disposition) is described in three categories: treated/released, transferred/hospitalized, and observed/left against medical advice (AMA)/unknown. NEISS data are based on a national probability sample and sample weights are summed to provide national estimates; valid regional and state-level estimates cannot be obtained from these data.

Age-adjusted and crude rates per 100,000 were calculated using U.S. Census bridged-race population estimates. To derive average annual estimates of nonfatal firearm-related injuries, weighted data ${ }^{3}$ for each year during 2010-2012 were summed and divided by 3 . To calculate annualized rates, the estimates were summed for the 3 years, then divided by the sum of the population estimates for the same period and multiplied by 100,000. Similar calculations were made to derive average annual number of deaths using unweighted data and annualized mortality rates. Case fatality rates were calculated by summing fatal and nonfatal cases within intent (e.g., homicide and assault; suicide and self-harm) and dividing the fatal cases in each intent category by the sum to determine the proportion of firearm injury cases within the given intent resulting in death. SAS and Joinpoint regression analyses ${ }^{4}$ were used to test the significance of trends across the 20-year period from 1993 to 2012. Annual Percent Change (APC) estimates that were statistically significant at $\mathrm{p}<0.05$ are presented to indicate the magnitude and direction of significant trends in age-adjusted firearm injury rates for each segment or period as determined by SAS and Joinpoint regressions.

\footnotetext{
${ }^{1}$ Firearm deaths were defined as all deaths of residents of the United States with one of following underlying cause of death codes from the International Classification of Diseases, 10th Revision: W32-W43 (unintentional firearm deaths), X72-X74 (firearm suicides), X93-X95 (firearm homicides), Y22-Y24 and U01.4 (firearm deaths of undetermined intent), and Y35.0 (legal intervention deaths by firearm). From 1993 to 1998, the corresponding ICD-9 codes were used to classify firearm deaths: unintentional firearm deaths (E922.0-E922.9), suicide or self-inflicted firearm injury deaths (E955.0-E955.4), assault-related firearm injury (E965.0E965.4), legal intervention injuries by firearm(E970), firearm injuries of undetermined intent (E985.0-E985.4).

${ }^{2}$ Unintentional firearm injury data for NEISS included $n=13,561$ missing race/ethnicity observations, approximately $20 \%$ of the total $\mathrm{N}$.

${ }^{3}$ NEISS data are weighted by size of hospital for all participating hospitals.

${ }^{4}$ Joinpoint regression analysis is a statistical method that examines successive segments of time, and the amount of increase or decrease within each segment to describe changing trends. A series of joined straight lines are fitted to the age-adjusted rates and the best-fitting point or points (joinpoints) are chosen, where the rate of increase or decrease is statistically significant.
} 
Cost estimation methods for CDC's WISQARS Cost of Injury module are described in detail elsewhere (Lawrence and Miller, 2014). Lifetime medical cost estimates include the cost of initial ED visits and hospitalizations for firearm injuries, and attributable lifetime medical costs (e.g., follow-up ED visits and hospitalizations, ambulance transportation, ambulatory care, prescription drugs, home health care), and nursing home and insurance claims administration costs. Loss of work estimates include lost expected employment earnings, lost fringe benefits, and lost value of household work. Medical costs were estimated from 2010 U.S. dollars (USD) data and inflated to 2012 USD using the U.S. Bureau of Economic Analysis Price Indexes for Personal Consumption Expenditures by Function. Work loss estimates for productivity loss are based on the U.S. Bureau of Labor Statistics' Employment Cost Index, Total Compensation and are reported in 2012 USD (Lawrence and Miller, 2014).

Data were analyzed using SAS, version 9.3 (SAS Institute, Inc.) and Joinpoint, version 4.1.0 (Statistical Methodology and Applications Branch, Surveillance Research Program, National Cancer Institute), software.

\section{Results}

\section{The extent of firearm injuries and deaths in the U.S}

On average, from 2010 to 2012 , more than 32,000 people $(n=32,529)$ died each year in the U.S. from a firearm-related injury, for an annual age-adjusted rate of 10.2 per 100,000 (Table 1). Sixty-two percent of these were suicides $(n=20,012), 35 \%$ were homicides $(n=$ $11,256)$, and $2 \%$ were unintentional firearm deaths $(n=582)$. The annual rate of firearm suicide was about twice as high as the annual rate of firearm homicide (7.2 vs 3.7) and about 38 times the annual rate of unintentional deaths from firearms (0.19).

During the same period of time, 67,197 people each year received medical treatment in an emergency department for a firearm-related injury from an assault, act of self-harm, or unintentionally, for an average annual age-adjusted rate of 21.6 per 100,000 people (Table 2). More than half of these cases resulted in hospitalization ( $n=36,224$ or 53.9\%) and about $43 \%$ were treated and released $(n=28,925)$. The remaining cases were observed in the ED or left against medical advice $(\mathrm{n}=2,049 ; 3 \%)$.

Unlike most causes of injury where deaths comprise a fraction of the total burden of injury, the average annual distribution of firearm-related deaths (33\% of the total) to hospitalizations (37\%) to emergency department visits (32\%) is similar, forming more of an injury tower than a pyramid (Fig. 1). This, in part, reflects the seriousness and lethality of firearm injuries. Firearm injuries are among the most lethal of health events. The case fatality rate (i.e., the proportion of cases resulting in death), however, varies by intent. Firearm-related self-harm has the highest case fatality rate. From 2010 to 2012, the average annual case fatality rate was $85 \%$ for firearm-related self-harm, 19\% for firearm-related assaults, and $5 \%$ for unintentional firearm injuries. 


\section{Who is at risk for a firearm-related injury?}

Rates of fatal and nonfatal firearm injuries are not distributed equally in the population. Age, gender, and race/ethnicity are among some of the factors that distinguish population groups most at risk of a firearm injury.

\section{Fatal firearm injuries}

Males disproportionately bear the burden of firearm mortality, accounting for $86 \%$ of all victims of firearm death. The annual rate of firearm death for males from 2010 to 2012 was 6.5 times higher than the annual rate for females $(18.1$ versus 2.8 per 100,000) (see Table 1). During this period, the annual rate ratio of the male firearm suicide rate to the female firearm suicide rate was 7:1, while the ratio of the male to female firearm homicide rate was about 5:1. As with firearm-related homicides and suicides, the large majority of victims of unintentional firearm deaths were males, with a male to female rate ratio of 6:1.

Young adults between the ages of 25 and 34 years have the highest rate of fatal firearm injury $(15.1$ per 100,000) of all age groups, followed by those in the 15 to 24 year age group (14.4) (see Table 1). Children under the age of 15 have the lowest fatal firearm injury rates (0.6 per 100,000). The overall rates, however, mask important patterns by intent. Rates of firearm suicide, for example, tend to increase with age. From 2010 to 2012, the annual rate of firearm suicide was highest among persons aged 65 years and older (10.9 per 100,000) followed by those in the 55-64 year age group (9.4) and the 45-54 year old age group (9.2). Rates of firearm homicide, on the other hand, are highest among adolescents and young adults and tend to decrease with age. From 2010 to 2012, the annual rate of firearm homicide was 8.9 per 100,000 among youth 15-24 years of age and 8.0 among those 25-34 years of age. Both age groups had rates of firearm homicide that were 1.8 to 10 times higher than those over 34 years of age. Both of these age groups also had the highest rates of unintentional firearm deaths ( 0.3 and 0.2 per 100,000, respectively). Children under the age of $15 \mathrm{had}$ the lowest rates of unintentional firearm deaths of all age groups (0.1).

Non-Hispanic blacks have the highest rates of firearm mortality overall (18.1 per 100,000), and this disparity is largely a function of differences between racial/ethnic groups in firearm homicide. From 2010 to 2012, the annual firearm homicide rate for non-Hispanic blacks (14.8 per 100,000) was about 15 times higher than the rate for the non-Hispanic Asian/ Pacific Islander group (1.0), 10.3 times higher than the rate for non-Hispanic whites (1.4), and 4.0-4.5 times higher than the rate for non-Hispanic American Indian/Alaskan native and Hispanic groups (3.7 and 3.3 per 100,000, respectively). The racial/ethnic differences in firearm homicide rates are especially pronounced among the younger age groups. For instance, in 2012 (data not shown), non-Hispanic black youth aged 15-24 years had a firearm homicide rate $(38.7$ per 100,000) that was 5 times that of their Hispanic counterparts (7.8 per 100,000) and more than 19 times the rate of their non-Hispanic white counterparts (2.0 per 100,000) (Centers for Disease Control and Prevention, National Center for Injury Prevention and Control, 2005).

In contrast to patterns of firearm homicide, non-Hispanic whites $(9.2$ per 100,000) and nonHispanic American Indian/Alaskan Native populations $(7.8$ per 100,000) have the highest 
rates of firearm suicide in the United States when compared to other groups. From 2010 to 2012, the annual rate of firearm suicide among non-Hispanic whites was 2.9-3.7 times higher than the rate for non-Hispanic black and Hispanic groups, and was 6.3 times higher than the rate for the non-Hispanic Asian/Pacific Islander group. Non-Hispanic white males account for the majority of firearm suicides. In 2012, for example (data not shown), $75 \%$ of all firearm suicides were among white males, with the highest rates occurring among those aged seventy years and older (35.3 per 100,000). From 2010 to 2012, rates of unintentional firearm deaths were highest among non-Hispanic American Indian/Alaskan Native populations (0.3), non-Hispanic blacks (0.2), and non-Hispanic whites (0.2). The nonHispanic Asian/Pacific Islander population had the lowest rates of unintentional firearm deaths (0.02).

\section{Non-fatal firearm injuries}

Similar to fatal firearm injuries, males bear the burden of nonfatal firearm injuries treated in U.S. emergency departments (ED), accounting for about $90 \%$ of all nonfatal firearm injuries medically treated each year. From 2010 to 2012, the average annual rate of nonfatal firearm injuries for males was 38.4 per 100,000 - or about 8.3 times the rate for females (4.6). Most of these injuries were from a firearm-related assault or were unintentional. This is largely due to the high case fatality rate for self-harm injuries involving a firearm. For both firearm assaults and unintentional firearm injuries, rates for males were about 9 times higher than those for females ( 27.9 vs. 3.2, for firearm assaults, and 6.6 vs. 0.7 for unintentional firearm injuries).

Young people under the age of 35 accounted for $72 \%$ of all nonfatal firearm injuries treated in U.S. emergency departments each year from 2010 to 2012. Most of these injuries resulted from a firearm-related assault and disproportionately impacted young people 15 to 34 years of age. The overall average annual rate of nonfatal firearm injuries was 65.6 per 100,000 among persons 15-24 years of age, and 44.2 among young adults 25-34 years of age. These age groups also had the highest rates of nonfatal unintentional firearm injury. Similar to firearm deaths, children under the age of 15 had the lowest rate of unintentional firearm injury across all age groups.

\section{Where do most firearm deaths occur?}

Patterns of firearm mortality vary by region in the United States (Table 1). From 2010 to 2012, nearly half of all firearm deaths occurred in the South for an overall annual rate of 12.6 per 100,000 . The Northeast region had both the lowest percentage and rate of firearm death of all regions in the U.S. (11\%; 6.4 per 100,000). By intent, the annual rates of firearm homicide (4.5), firearm suicide (8.8) and unintentional firearm death (0.3) were all higher in the South when compared to other regions of the United States. Next to the South, rates of firearm suicide were also higher in the West compared to other regions (7.6 versus 3.9 in the Northeast and 6.9 in the Midwest). Rates of unintentional firearm deaths in the Northeast, Midwest, and Western regions of the United States were similar (0.1 per 100,000). 


\section{Trends}

Patterns of firearm injuries and deaths have changed over time. Trends in age-adjusted firearm death and injury rates between 1993 and 2012 were analyzed and overall firearm death and injury trends are depicted in Fig. 2. Nonfatal firearm injury rates declined sharply from 1993 to 1999, decreasing 50\% from 38.3 to 19.3 (Annual Percentage Change (APC) = $-10.5, P<.05)$, and then remained relatively stable. Firearm death rates exhibited a smaller but significant decline from 1993 to 1999, decreasing 31\% from 15.0 to 10.3 (APC $=-6.3$, $P<.05$ ), and then remained relatively stable from 1999 to 2012, exhibiting no further significant change.

Although overall firearm death and injury rates remained stable between 1999 and 2012, these findings mask different trends by injury intent (Fig. 3). Firearm homicide rates showed two significant periods of decline, decreasing $48 \%$ from 6.8 to 3.8 between 1993 and 1999 (APC $=-9.4, P<.001$ ), and decreasing 12\% from 4.3 to 3.8 between 2006 and 2012 (APC $=-2.7, P<.001)$. Firearm suicide rates significantly declined between 1993 and 1999, decreasing $20 \%$ from 8.6 to 6.9 (APC $=-3.3, P<.05$ ), and between 1999 and 2006, decreasing $6 \%$ from 6.9 to 6.5 ( $\mathrm{APC}=-1.2, P<.05)$. However, firearm suicides have recently increased significantly again, going up 17\% from 6.5 to 7.6 between 2006 and 2012 $(\mathrm{APC}=2.1, P<.05)$. Unintentional firearm death rates also showed two significant periods of decline, decreasing 50\% from 0.6 to 0.3 between 1993 and 1999 (APC $=-10.9, P<.05$ ), and decreasing $33 \%$ from 0.3 to 0.2 between 1999 and 2012 (APC $=-3.8, P<.05$ ).

Similar to firearm homicide rates, assault-related nonfatal firearm injury rates significantly declined between 1993 and 1999 (Fig. 4), decreasing 52\% from 23.5 to 11.3 (APC $=-11.9$, $P<.05)$. Following this period of decline, nonfatal firearm assault injury rates increased significantly between 1999 and 2012, increasing 52\% from 11.3 to 17.1 (APC $=2.7, P<$. 05). Unintentional nonfatal firearm injury rates significantly declined between 1993 and 1999, decreasing 54\% from 6.9 to 3.2 (APC $=-11.0, P<.05$ ), and then remained relatively stable from 1999 to 2012, exhibiting no further significant change.

\section{Injury characteristics and healthcare response}

Firearm injuries often require a medical response. This response varies depending on the nature and severity of the firearm injury and can range from acute care treatment in a clinic or hospital emergency department to a hospital stay or more long-term care (e.g., rehabilitation following a spinal cord or brain injury). From 2010 to 2012, more than $70 \%$ of persons with a nonfatal firearm injury were taken to a medical facility for treatment either by EMS/ambulance or by air transport (Table 2). This was true for most cases, except for unintentional firearm injuries, where a sizeable proportion also arrived by private vehicle $(30 \%)$.

Persons arriving for medical treatment for a firearm injury due to unintentional circumstances frequently had leg and foot injuries (43\%), followed by injuries to their arm or hand (34\%) (Fig. 5). Persons arriving for treatment following a firearm assault also frequently had leg and foot injuries (35\%). Gunshot wounds to the upper and lower trunk, however, were more common among assault cases than unintentional firearm injury cases 
( $20 \%$ vs. $6 \%$ for upper trunk injuries; $19 \%$ vs. $7 \%$ for lower trunk injuries). The percentage of assault or unintentional cases with gunshot wounds to the head or neck was about the same (11\% and $10 \%$, respectively). It is important to note that gunshot wounds to the head or neck are often fatal—only about a third of patients with these types of wounds survive long enough to arrive at the hospital (Blissitt, 2006).

The majority of cases presenting with leg/foot injuries or arm/ hand injuries, regardless of intent, were treated and released (Table 3). In contrast, injuries to the upper and lower trunk often resulted in hospitalization. This was true for both assault cases and unintentional firearm injury cases. With assault-related cases, the majority of firearm injuries to the head or neck also resulted in hospitalization (66\%). With unintentional firearm injury, the number of patients with head or neck injuries was too small to produce reliable estimates by disposition.

The medical and work loss costs associated with firearm injuries are substantial. Using average annual frequencies between 2010 and 2012, firearm deaths and injuries resulted in over $\$ 48$ billion in combined lifetime medical and work loss costs (estimate:

$\$ 48,292,384,000)$. Ninety-one percent of these costs were attributed to fatal firearm injuries $(\$ 44,041,023,000)$. The majority of costs for each of the three dispositions (died, hospitalized, treated and released) were work loss costs; however, the percentages differed for each. Ninety-nine percent of fatal firearm injury costs were attributed to work loss, while $79 \%$ (hospitalized) and 61\% (treated and released) were attributed to work loss for the nonfatal firearm injury groups. The composition of costs varied by intent within each disposition as well, with self-harm/suicide resulting in the greatest costs for fatal firearm injuries, and assault/homicide resulting in the greatest costs for nonfatal firearm injuries (see Fig. 6).

\section{Discussion}

The findings in this paper highlight the magnitude and impact of firearm violence in the United States. For every 100,000 people in the U.S. who die in an act of firearm violence, about the same number die in motor vehicle crashes (about 10 per 100,000). Apart from being a common problem, many Americans may not realize that over $60 \%$ of all firearm deaths in the U.S. are suicides-almost double the number of firearm homicides. This reflects, in part, the lethality of firearms particularly when used to attempt suicide. About $85 \%$ of people who use a firearm to attempt suicide die from their injury-in contrast to about $20 \%$ of persons injured in firearm-related assaults. Other methods used in suicide attempts tend to have much lower case fatality rates (e.g., cut/pierce injuries, $0.7 \%$; poisoning, 2.5\%; jumping/falling, $\left.19.9 \%^{5}\right)($ Centers for Disease Control and Prevention, National Center for Injury Prevention and Control, 2005). Given that suicides may be impulsive (Simon et al., 2001), the lethality of the method selected can be a critical determinant of whether the attempt is fatal or nonfatal. Previous research indicates that the time between deciding on suicide and attempting suicide can be as little as 10 min or less (Simon et al., 2001; Deisenhammer et al., 2009). There is also some evidence to suggest that

\footnotetext{
${ }^{5}$ However, strangulation/suffocation, which includes hanging, is an exception. It also has a high case-fatality rate, around $70 \%$.
} 
more people start an attempt and then stop mid-way than carry it through to completion (Drum et al., 2009). Unlike firearms or jumping from a tall bridge or building, methods such as cutting, poisoning, and overdose offer a window of opportunity for rescue. It is also important to note that fewer than $10 \%$ of persons who non-fatally attempt suicide go on to die by suicide (Owens et al., 2002). Previous research also indicates that people do not substitute a different method when a highly lethal method is unavailable or difficult to access (Hawton, 2007). The lethality of the method available during an acute suicidal crisis can therefore make an important difference in the outcome.

The impact of firearm violence, however, extends well beyond deaths. Nearly 70,000 people suffer nonfatal gunshot wounds each year. This includes people hospitalized with serious injuries-most often from gunshot wounds to the trunk. Previous studies have shown that nonfatal firearm injury is a leading cause of spinal cord injuries in the United States (Hemenway and American society, 2006), and that these injuries are more likely to result in paraplegia than other types of spinal cord injuries (McKinley et al., 1999). Many people hospitalized with non-fatal gunshot wounds experience long-term consequences, including physical disabilities (DiScala and Sege, 2004) and chronic mental health problems from conditions such as post-traumatic-stress disorder (Greenspan and Kellermann, 2002). Others are treated and released from emergency departments with wounds to their extremities, and experience shorter term or less severe physical impairment.

Firearm violence affects people in all stages of life, but disproportionately impacts males, younger age groups (with the exception of firearm suicide), and racial/ethnic minorities. Firearms have figured prominently in assaults, crime, and homicide involving young males for decades (Blumstein, 2002; Dahlberg and Potter, 2001; Reiss and Roth, 1993). Even though rates of firearm homicide among youth aged 15-24 have declined noticeably since their peak in 1993, the proportion of youth homicides committed with firearms has remained consistently high over time-with a range in annual percentages of 80-91\% (Centers for Disease Control and Prevention, National Center for Injury Prevention and Control, 2005; Dahlberg and Potter, 2001). The age groups with the highest rates of firearm homicide (those aged 15-34 years), are also the age groups with the highest rates of unintentional firearm injuries. This may be due to inexperience handling firearms, situational factors (e.g., showing a gun to others, playing with a gun) or a byproduct of weapon carrying and use (e.g., hunting, target shooting). Younger children are also impacted by unintentional firearm injuries, but have the lowest rates. In part, this could be due to safe storage practices in homes with children. Previous research shows that young children are curious about firearms and will even touch a firearm when instructed not to do so (Hardy et al., 1996) which points to the need to store firearms safely and out of reach of young children.

Males also bear a majority of the burden of self-directed firearm violence. Rates of suicide in the U.S. are higher among males than females. Females have higher rates of non-fatal suicide attempts. This difference is largely due to the lethality of methods used by men and women. Firearms and hanging/suffocation are the most common methods used by men to attempt suicide (Centers for Disease Control and Prevention, National Center for Injury Prevention and Control, 2005). Women tend to use less lethal methods such as poisoning, although their rates of suicide by firearm and hanging/suffocation have increased in recent 
years (Centers for Disease Control and Prevention, National Center for Injury Prevention and Control, 2005; Sullivan et al., 2013).

Ethnic minority populations are also especially impacted by firearm violence. Life expectancy for black males, for instance, is on average, 5 years lower than that of white males, a difference that is due in part to homicide (Kochanek et al., 2013). Firearm homicides, in particular, account for nearly $14.5 \%$ of years of potential life lost before age 65 among black males compared to 1.2\% among white males (Centers for Disease Control and Prevention, National Center for Injury Prevention and Control, 2005). There are many factors that potentially mediate and moderate the risk for firearm violence. These include growing up in environments characterized by concentrated disadvantage, school failure, drug and firearm trafficking, exposure to gangs, neighborhood disorder, witnessing violence, involvement with weapons, and poor family functioning and instability (Reiss and Roth, 1993; Farrington et al., 2012; Loeber and Farrington, 2011; Morenoff et al., 2001; Sampson et al., 1997; Molnar et al., 2004; Dahlberg and Simon, 2006; American Psychological Association, 2013). Even within these communities, firearm violence is not evenly distributed by geography or among the populations living in these communities. Rather it is highly concentrated in specific "hot spot" locations and often occurs within high-risk social networks ("co-offending networks") (Braga et al., 2009). For example, one study found that $74 \%$ of the gun violence in Boston over a 29-year time frame occurred in only $5 \%$ of the street blocks and intersections in the city (Braga et al., 2009). The "high-risk social networks" also comprised a relatively small percentage of the population within these neighborhoods. For example, $85 \%$ of the injuries from firearm violence in one Boston community were experienced within one social network, comprising only $5 \%$ of the neighborhood population (Papachristos et al., 2012; Papachristos and Wildeman, 2014). Involvement in or proximity to these "high-risk social networks" is, therefore, an important risk factor for firearm injury and death above and beyond other factors.

The findings in this paper also highlight a number of notable trends in rates of firearm violence in the United States. From 1993 to 1999, rates of firearm violence declined significantly - a pattern seen for both fatal and nonfatal firearm violence and across all types of firearm violence. The steep decline in firearm homicides during this time has been described elsewhere (Blumstein, 2002; Blumstein and Wallman, 2006; Rosenfeld, 2002). Previous research points to several potential contributing factors including the cycling up and down of youth firearm homicides (more so than adult homicides), changes in markets for illegal drugs (particularly the crack cocaine market which swept across urban cities in the 1980s and crested about 1990), changes in juvenile arrest policies and penalties for drugrelated crime in the Violent Crime Control and Law Enforcement Act of 1994, improved economic conditions, and an increase in community-based policing strategies and primary prevention strategies for youth, families, schools and communities (Blumstein, 2002; Blumstein and Wallman, 2006; Rosenfeld, 2002; Braga and Weisburd, 2011; Ferdon et al., 2013). Firearm homicides declined in all regions during this period of time mirroring the overall trends (Planty and Truman, 2013).

Our findings also point to a substantial drop in nonfatal firearm assaults during the same period that firearm homicides declined. The pattern for fatal and nonfatal interpersonal 
violence, however, diverged in the period since 2000. Whereas rates of firearm homicide remained relatively flat from 2000 to 2012 (with an uptick in 2006 and another in 2012), rates of nonfatal firearm assaults trended upward. By 2012, rates of medically treated nonfatal firearm assaults were at their highest level since 1995. Nevertheless, the recent upward trend in nonfatal firearm injury rates should be interpreted with caution until national and state nonfatal firearm injury data from other data sets (e.g., state-based hospital discharge and ED data) becomes available to examine these findings further. Other indicators such as arrests for violent crime exhibit a pattern similar to firearm homicide from 2002 to 2012 (Department of Justice and Federal Bureau of Investigation, 2000-2013). The juvenile violent crime index arrest rate also shows an increase in the mid-2000s, and then a decline through 2012 to its lowest level since at least 1980 (Office of Juvenile Justice and Delinquency Prevention, 1980-2012). These trends suggest a change in both absolute levels of violent crime as well as in the lethality of the violence.

Advances in the medical treatment of firearm injuries may be keeping more persons with firearm injuries alive than was the case previously. The relationship between lower lethality of violent assaults and improved medical treatment has been posited since the post-World War II era, and the continuous drop in lethality since 1960 has been attributed to factors such as faster response time to violent incidents, improved rapid emergency transportation, and advances in medical treatment of violent injuries. Using Uniform Crime Reports and vital statistics national data, Harris et al. (2002) found that indicators of urbanization and access to intensive medical care such as number of hospitals, hospital-affiliated physicians, and hospitals with open heart surgery facilities were all significantly associated with lower lethality of violent assaults between 1976 and 1997. Lower case-fatality rates over time for firearm assaults were evident in our data as well. After a case fatality rate high of $27 \%$ in 2002 , the case fatality rate for firearm homicide/assaults reached their lowest point of $18 \%$ by 2012 .

Firearm suicides also declined during the 1990s, although not as steeply as those for firearm homicide or nonfatal firearm assaults. Improved economic conditions may partly explain the drop in firearm suicides during this period. Previous research indicates that suicide tends to track with business cycles, decreasing during periods of economic expansion and increasing during times of economic recession (Luo et al., 2011). The national rate of unemployment declined from 6.9 in 1993 to 4.0 in 2000-the lowest it had been since 1969. In contrast, the uptick in firearm suicides beginning in 2007 may be related to the recent economic downturn. National rates of unemployment doubled between 2006 and 2010 increasing from 4.6 in 2006 to 9.6 in 2010 and then dropped to 8.1 by 2012 (Department of Labor and Bureau of Labor Statistics, 1944 to date). Reports have also pointed to a significant increase in suicide among middle-aged adults from 1990 to 2010 (Sullivan et al., 2013)—an age group hard hit by the economic downturn but also a cohort that historically has had high rates of suicide (Phillips et al., 2010).

In contrast to the findings for interpersonal and self-directed firearm violence, unintentional firearm injuries have been trending downward since the 1920s (Frattaroli et al., 2002; Ikeda et al., 1997). The continuation of this downward trend is evident in both the fatal and nonfatal data for the periods examined here. Previous research points to possible changes in 
exposure-e.g., changes in household firearm ownership, safety practices, shifting demographics, and motivations for owning or possessing a firearm (Frattaroli et al., 2002). Another possible explanation relates to changes in cause-of-death coding practices for selfinflicted injuries over time (e.g., shifts over time in the pressures placed on coroners/medical examiners by families of decedents to classify self-inflicted firearm deaths as unintentional rather than as suicide due to religious taboos, social stigma, and concerns about loss of life insurance benefits (Hanzlick and Combs, 1998)).

The findings in this paper are subject to a few limitations. First, while the data sources are well-suited for capturing the burden and epidemiological profile of firearm injury and death in the U.S., they are limited in the extent to which they offer information about the context and circumstances surrounding firearm violence, including information about the relationship of the victim to the perpetrator. There are other datasets more suited to a detailed analysis of firearm injury incidents, such as the National Violent Death Reporting System (NVDRS). NVDRS, however, is more limited in its coverage of the U.S. and years of available data. Second, there is potential misclassification of certain racial/ethnic groups (e.g., Hispanics, Asian/Pacific Islanders, and American Indian/Alaskan Natives) in death certificate data (Arias et al., 2008). Estimates derived from death certificate data may therefore underestimate victimization in these groups. The extent of missing data on race and ethnicity in NEISS precluded an examination of nonfatal firearm injuries by race/ ethnicity. Therefore, it is difficult to know whether the patterns of nonfatal firearm victimization mirror those for fatal victimization among the different racial/ethnic groups. Third, the coding of mortality changed substantially in 1999 as the National Center for Health Statistics transitioned from the ICD-9 system to ICD-10. While the new coding system impacted mortality coding for some leading causes of death, the comparability ratios for intentional self-harm(suicide) and assault (homicide) were very close to 1.0 suggesting that the revision did not substantially affect mortality patterns for suicide or homicide (Anderson et al., 2001). Fourth, WISQARS Cost of Injury estimates are limited to medical and work loss costs, and do not capture broader social costs of firearm violence discussed by others (Cook and Ludwig, 2002; Hemenway, 2011), such as property values, tax expenditures, costs incurred by persons other than one who sustained injuries (such as family members), and lost quality of life. As a final limitation, nonfatal firearm injuries treated in settings other than hospital emergency departments and inpatient settings, or which are not medically treated at all are not included in this study.

\section{Conclusion}

Firearm injuries are an important public health problem in the United States contributing substantially each year to premature death, illness, and disability. The human toll is only part of their devastating impact. The economic impact of firearm-related deaths and injuries costs the United States nearly $\$ 50$ billion each year in medical and lost productivity costs alone. Understanding the nature, magnitude and health impact of firearm violence is only the first step in preventing firearm violence. Finding ways to prevent such injuries remains one of the most important goals and challenges of public health. As noted in the recent report from the Institute of Medicine/National Research Council (Institute of Medicine/National Research Council, 2013), achieving measurable reductions in firearm violence remains a more elusive 
challenge yet is also attainable with research to better understand risk and protective factors and the strategies that effectively prevent firearm violence.

\section{References}

American Psychological Association. Gun violence: prediction, prevention, and policy. 2013 Retrieved from. http://www.apa.org/pubs/info/reports/gun-violence-prevention.aspx.

Anderson, RN.; Minino, AM.; Hoyert, DL.; Rosenberg, HM. National Vital Statistics Reports. Vol. 49. Hyattsville, Maryland: National Center for Health Statistics; 2001. Comparability of cause of death between ICD-9 and ICD-10: preliminary estimates.

Arias E, Schauman WS, Eschbach K, Sorlie PD, Backlund E. The validity of race and Hispanic origin reporting on death certificates in the United States. Vital Health Stat. 2008; 2(148)

Blissitt P. Care of the critically ill patient with penetrating head injury. Crit. Care Nurs. Clin. North Am. 2006; 18(3):321-332. [PubMed: 16962454]

Blumstein R. Youth, guns, and violent crime. Future Child. 2002; 19(2):39-53.

Blumstein, A.; Wallman, J., editors. The Crime Drop in America, revised edition. New York, NY: Cambridge University Press; 2006.

Braga AA, Weisburd DL. The effects of focused deterrence strategies on crime: a systematic review and meta-analysis of the empirical evidence. J. Res. Crime Delinq. 2011; 49:323-358.

Braga AA, Papachristos AV, Hureau DM. The concentration and stability of gun violence at micro places in Boston, 1980-2008. J. Quant. Criminol. 2009; 26(1):33-53.

Centers for Disease Control and Prevention, National Center for Injury Prevention and Control. [2015 January 15] Web-based Injury Statistics Query and Reporting System (WISQARS) [online]. 2005. (Available from URL: www.cdc.gov/ncipc/wisqars).

Cook, PJ.; Ludwig, J. Gun Violence: The Real Costs. New York: Oxford University Press; 2002. (0-19-513793-0).

Dahlberg LL, Potter LB. Youth violence: developmental pathways and prevention challenges. Am. J. Prev. Med. 2001; 20(1S):3-14. [PubMed: 11146255]

Dahlberg, LL.; Simon, TR. Predicting and preventing youth violence: developmental pathways and risk. In: Lutzker, JR., editor. Preventing Violence: Research and Evidence-Based Intervention Strategies. Washington, DC: American Psychological Association; 2006. p. 97-124.

Deisenhammer EA, Ing CM, Strauss R, Kemmler G, Hinterhuber H, Weiss EM. The duration of the suicidal process: how much time is left for intervention between consideration and accomplishment of a suicide attempt? J. Clin. Psychiatry. 2009; 70(1):19-24. [PubMed: 19026258]

U.S. Department of Justice, Federal Bureau of Investigation. Crime in the United States, Annual Reports for Years 2000-2013. Available on the Internet: http://www.fbi.gov/about-us/cjis/ucr/ crime-in-the-u.s

U.S. Department of Labor, Bureau of Labor Statistics. e. Employment status of the civilian noninstitutional population, 1944 to date. Current Population Survey, Household Annual Averages, Table 1. Available on the Internet: http://www.bls.gov/cps/cpsaat01.pdf.

DiScala C, Sege R. Outcomes in children and young adults who are hospitalized for firearms-related injuries. Pediatrics. 2004; 113(5):1306-1312. [PubMed: 15121946]

Drum D, Brownson C, Denmark A, Smith S. New data on the nature of suicidal crises in college students: shifting the paradigm. Prof. Psychol. Res. Pract. 2009; 40(3):213-222.

Farrington DP, Loeber R, Berg MT. Young men who kill: a prospective longitudinal examination from childhood. Homicide Stud. 2012; 16(2):99-128.

Ferdon CD, Dahlberg LL, Kegler S. Homicide rates among persons aged 10-24 years-United States, 1981-2010. Morb. Mortal. Wkly Rep. 2013 Jul 12; 62(27):545-548.

Frattaroli S, Webster DW, Teret SP. Unintentional gun injuries, firearm design, and prevention: what we know, what we need to know, and what can be done. J. Urban Health. 2002; 79(1):49-59. [PubMed: 11937615] 
Greenspan AI, Kellermann AL. Physical and psychological outcomes eight months after serious gunshot injury. J. Trauma. 2002; 53(4):709-716. [PubMed: 12394871]

Hanzlick R, Combs D. Medical examiner and coroner systems: history and trends. JAMA. 1998; 279:870-874. [PubMed: 9516003]

Hardy MS, Armstrong FD, Martin BL, Strawn KN. A firearm safety program for children: they just can't say no. Dev. Behav. Pediatr. 1996; 17:216-221.

Harris AR, Thomas SH, Fisher GA, Hirsch DJ. Murder and medicine: the lethality of criminal assault 1960-1999. Homicide Stud. 2002; 6(2):128-166.

Hawton K. Restricting access to methods of suicide: rationale and evaluation of this approach to suicide prevention. Crisis. 2007; 28(1):4-9. [PubMed: 17555027]

Hemenway D. Measuring the cost of injury: underestimating the costs of street violence (commentary). Inj. Prev. 2011; 17:289-290. [PubMed: 21865206]

Hemenway, D.; Guns, American society. Private Guns, Public Health. 2nd. Ann Arbor, MI: University of Michigan Press; 2006. p. 13(978-0-472-02382-0).

Ikeda RM, Gorwitz R, James SP. Trends in fatal firearm-related injuries, United States, 1962-1993. Am. J. Prev. Med. 1997; 13(5):396-400. [PubMed: 9315274]

Institute of Medicine/National Research Council. Priorities for Research to Reduce the Threat of Firearm-Related Violence. Washington, DC: Institute of Medicine; 2013 Jun 5. Available on the Internet: http://www.iom.edu/Reports/2013/Priorities-for-Research-to-Reduce-the-Threat-ofFirearm-Related-Violence.aspx).

Kochanek, KD.; Arias, E.; Anderson, R. NCHS Data Brief, No 125. Hyattsville MD: National Center for Health Statistics; 2013. How did Cause of Death Contribute to Racial Differences in Life Expectancy in the United States in 2010 ?

Lawrence, BA.; Miller, TR. Medical and Work Loss Cost Estimation Methods for the WISQARS Cost of Injury Module. Calverton, MD: Pacific Institute for Research and Evaluation; 2014. Available from: http://www.cdc.gov/injury/wisqars/pdf/wisqars_cost_methods-a.pdf)

Loeber, R.; Farrington, DP. Young Homicide Offenders and Victims: Risk Factors, Prediction and Prevention from Childhood. New York, NY: Springer; 2011.

Luo F, Florence CS, Quispe-Agnoli M, Ouyang L, Crosby AE. Impact of business cycles on US suicide rates, 1928-2007. Am. J. Public Health. 2011; 101(6):1139-1146. [PubMed: 21493938]

McKinley WO, Johns JS, Musgrove JJ. Clinical presentations, medical complications, and functional outcomes of individuals with gunshot wound-induced spinal cord injury. Am. J. Phys. Med. Rehabil. 1999; 78(2):102-107. [PubMed: 10088582]

Molnar BE, Miller MJ, Azrael D, Buka SL. Neighborhood predictors of concealed firearm carrying among children and adolescents: results from the project on human development in Chicago neighborhoods. Arch. Pediatr. Adolesc. Med. 2004; 158:657-664. [PubMed: 15237065]

Morenoff JD, Sampson RJ, Raudenbush SW. Neighborhood inequality, collective efficacy, and the spatial dynamics of urban violence. Criminology. 2001; 39:517-559.

Office of Juvenile Justice and Delinquency Prevention. Juvenile Arrest Rates for Violent Crime Index Offenses, 1980-2012. OJJDP Statistical Briefing Book. Available on the Internet: http:// www.ojjdp.gov/ojstatbb/crime/JAR_Display.asp?ID=qa05201.

Owens D, Horrocks J, House A. Fatal and non-fatal repetition of self-harm. Systematic review. Br. J. Psychiatry. 2002; 181:193-199. [PubMed: 12204922]

Papachristos AV, Wildeman C. Network exposure and homicide victimization in an African American community. Am. J. Public Health. 2014; 104(1):143-150. [PubMed: 24228655]

Papachristos AV, Braga AA, Hureau DM. Social networks and the risk of gunshot injury. J. Urban Health. 2012; 89(6):992-1003. [PubMed: 22714704]

Phillips JA, Robin AV, Nugent CN, Idler EL. Understanding recent changes in suicide rates among the middle-aged: period or cohort effects? Public Health Rep. 2010; 125:680-688. [PubMed: 20873284]

Planty M, Truman JL. Firearm violence-1993-2011. Bureau of Justice Statistics, Department of Justice, NCJ 241730. 2013 (Available on the internet: http://www.bjs.gov/content/pub/pdf/ fv9311.pdf). 
Reiss, AJ., Jr; Roth, JA., editors. Understanding and preventing violence. Vol. 1. Washington, DC: National Academy Press; 1993.

Rosenfeld, R. Contexts. Sage Publications; 2002. Crime decline in context; p. 1-25.

Sampson RJ, Raudenbush SW, Earls F. Neighborhoods and violent crime: a multilevel study of collective efficacy. Science. 1997; 277:919-924.

Simon TR, Swann AC, Powell KE, Potter LB, Kresnow M, O'Carroll PW. Characteristics of impulsive suicide attempts and attempters. SLTB. 2001; 32((supp)):49-59.

Sullivan EM, Annest LL, Luo F, Simon TR, Dahlberg LL. Suicide among adults aged 35-64 yearsUnited States, 1999-2010. Morb. Mortal. Wkly Rep. 2013 May 3; 62(17):321-325.

U.S. Consumer Products Safety Commission. Bethesda, MD: U.S. Consumer Products Safety Commission; 2014. National Electronic Injury Surveillance System (NEISS). (Available from: http://www.cpsc.gov//Global/Neiss_prod/completemanual\%20.pdf). 


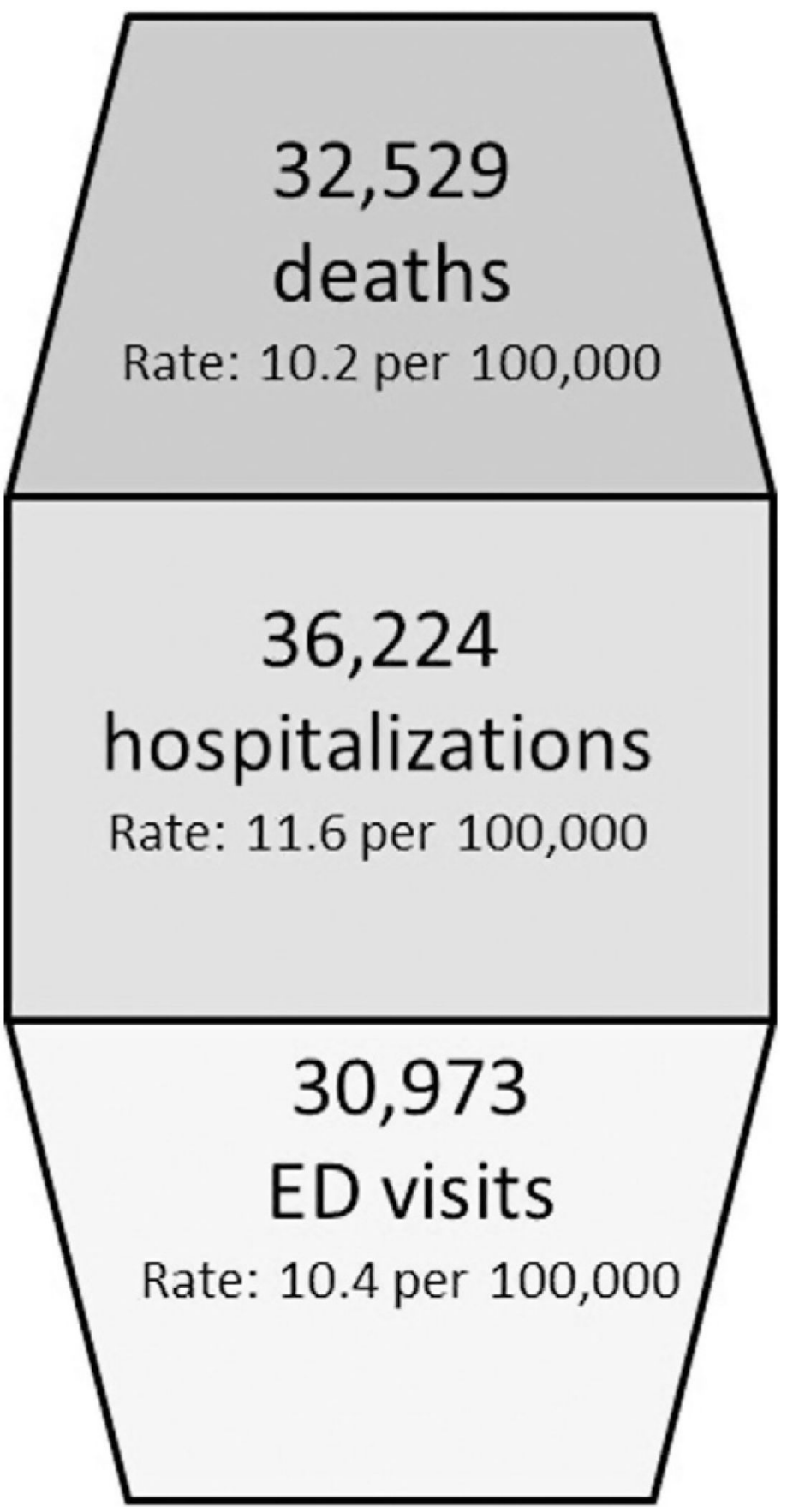

Fig. 1.

Distribution of medical outcome of firearm injuries, all persons-United States, 2010-2012. Data source: National Vital Statistics System for firearm injury deaths; CDC/National Electronic Injury Surveillance System; US Census Bureau for population estimates. Rates reported are age-adjusted rates per 100,000. Hospitalizations include persons categorized as hospitalized or transferred upon discharge from the emergency department, and emergency department visits include persons treated and released from the emergency department, or 
who were reported to have been observed, left against medical advice, or whose disposition was unknown after presenting to the emergency department. 


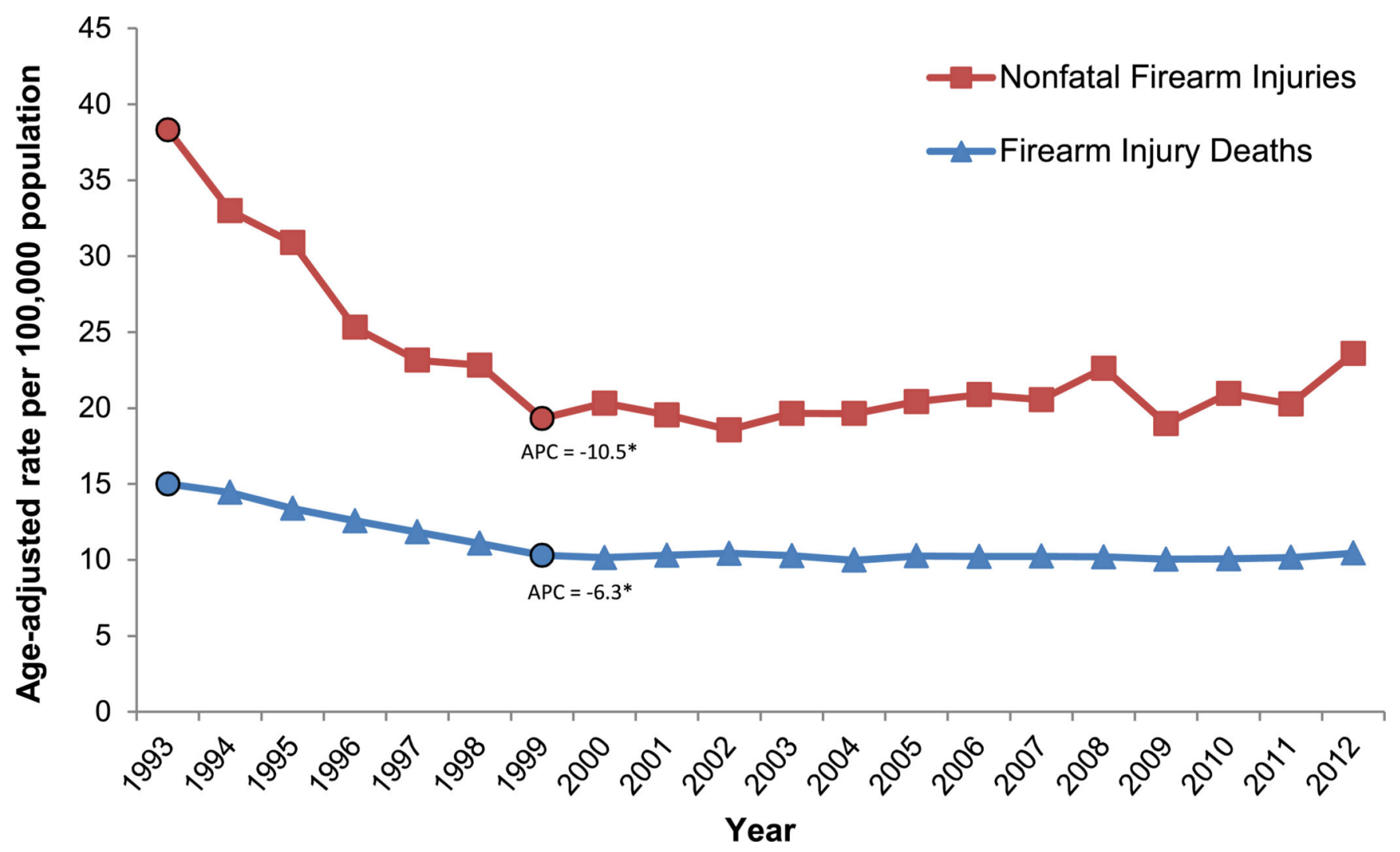

Fig. 2.

Age-adjusted fatal and nonfatal firearm injury rates by year, United States, 1993-2012. Data source: National Vital Statistics System for firearm injury deaths; CDC/National Electronic Injury Surveillance System (NEISS) for nonfatal firearm injuries; US Census Bureau for population estimates. APC $=$ Annual Percentage Change. Statistical significance of regression results indicated as $* P<.05$. 


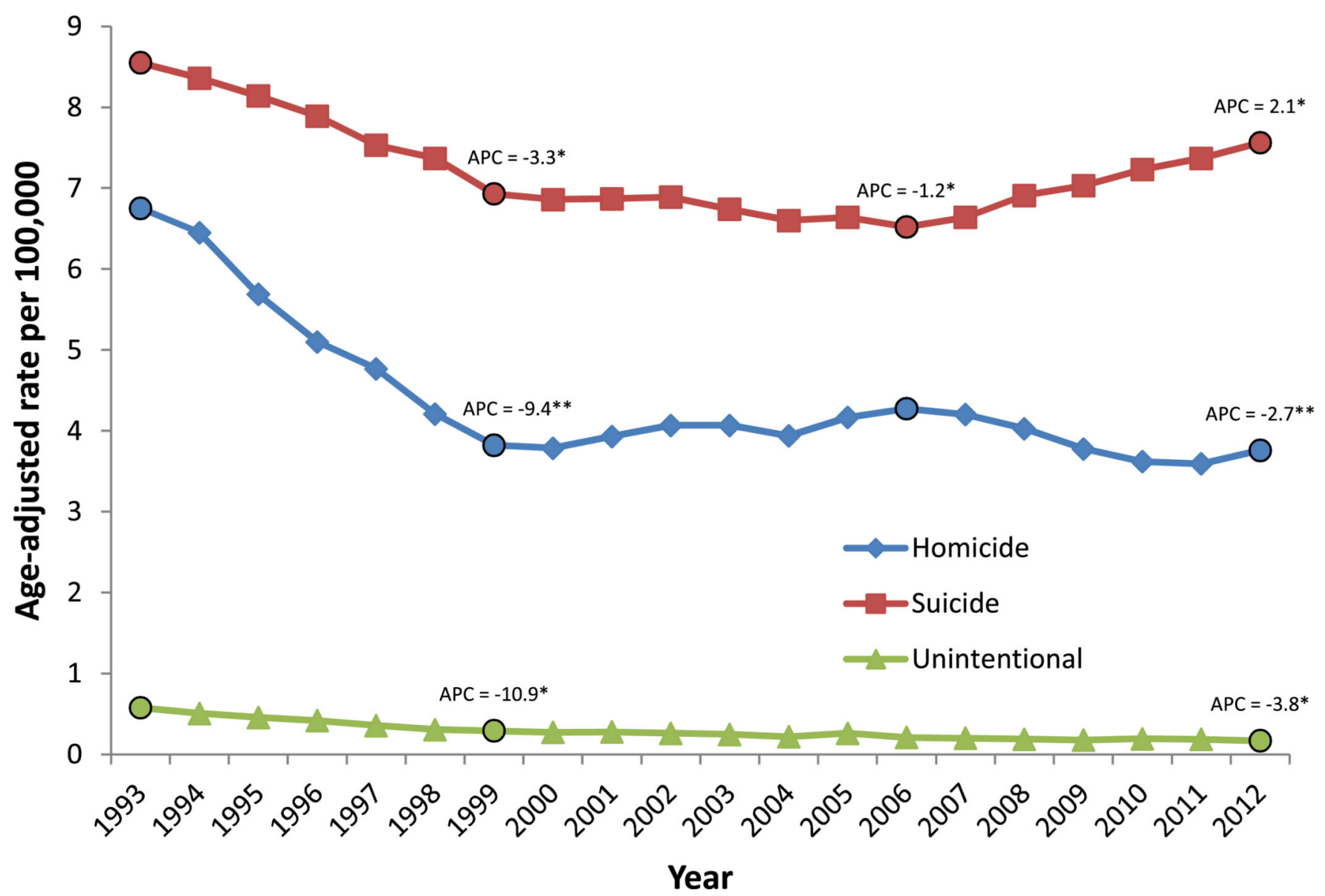

\section{Fig. 3.}

Age-adjusted fatal firearm injury rates by intent and year, United States, 1993-2012. Data source: National Vital Statistics System, US Census Bureau for population estimates. APC $=$ Annual Percentage Change. Statistical significance of regression results indicated as $* P<$. $05, * * P<.001$. Age-adjusted firearm suicide rates reflect rates for decedents 10 years of age and older. 


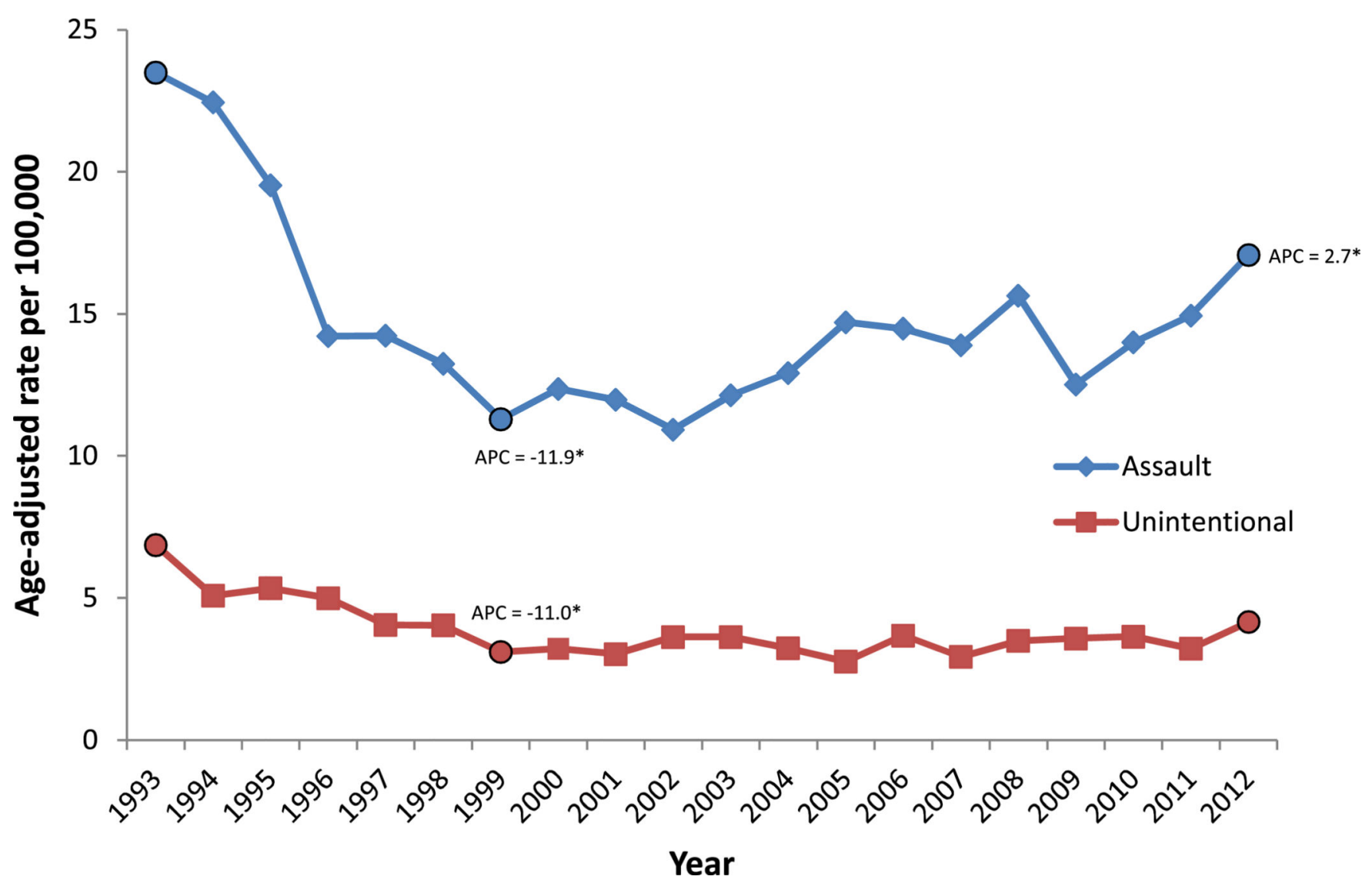

Fig. 4.

Age-adjusted nonfatal firearm injury rates by intent and year, United States, 1993-2012.

Data source: CDC/National Electronic Injury Surveillance System(NEISS); US Census

Bureau for population estimates. APC $=$ Annual Percentage Change. Statistical significance of regression results indicated as $* P<.05$. 


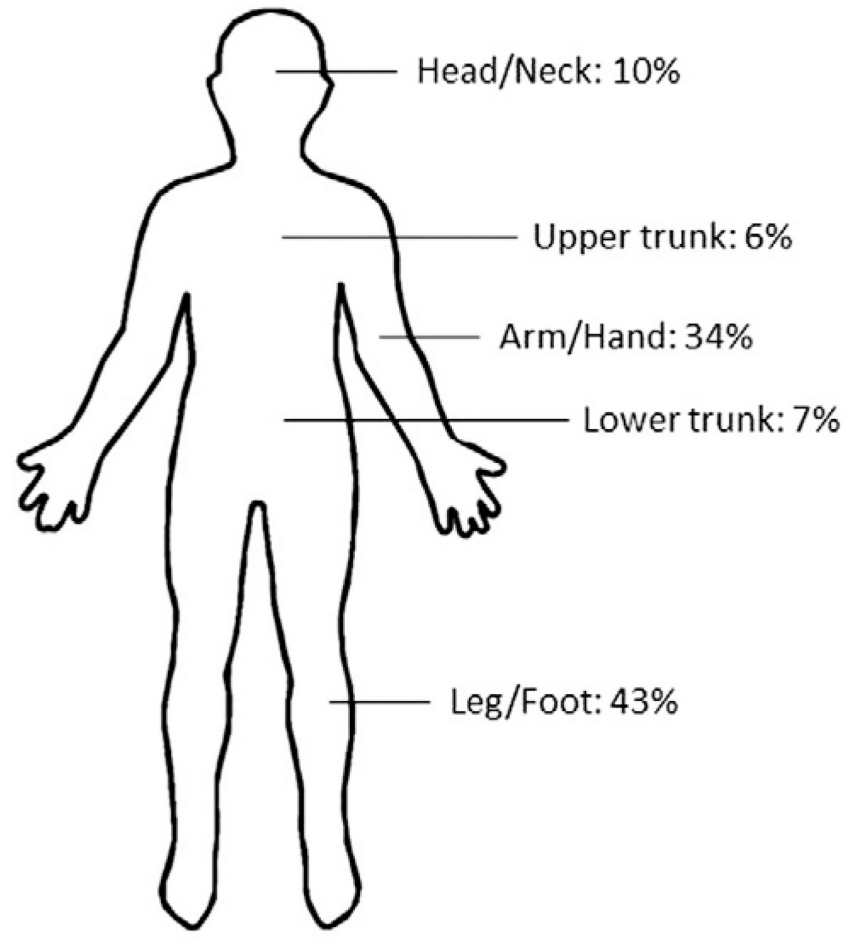

Unintentional

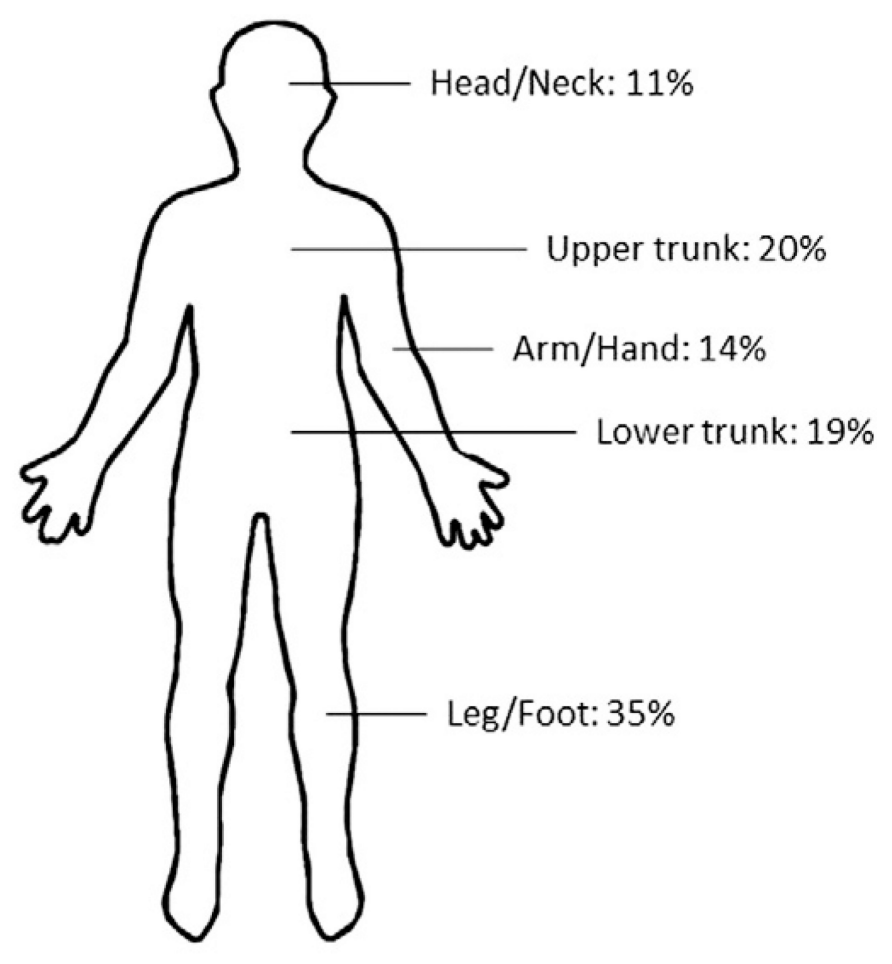

Assault

Fig. 5.

Primary body part affected by unintentional vs. assault-related nonfatal firearm injuries2010-2012. Data source: CDC/National Electronic Injury Surveillance System (NEISS); data obtained by request. 


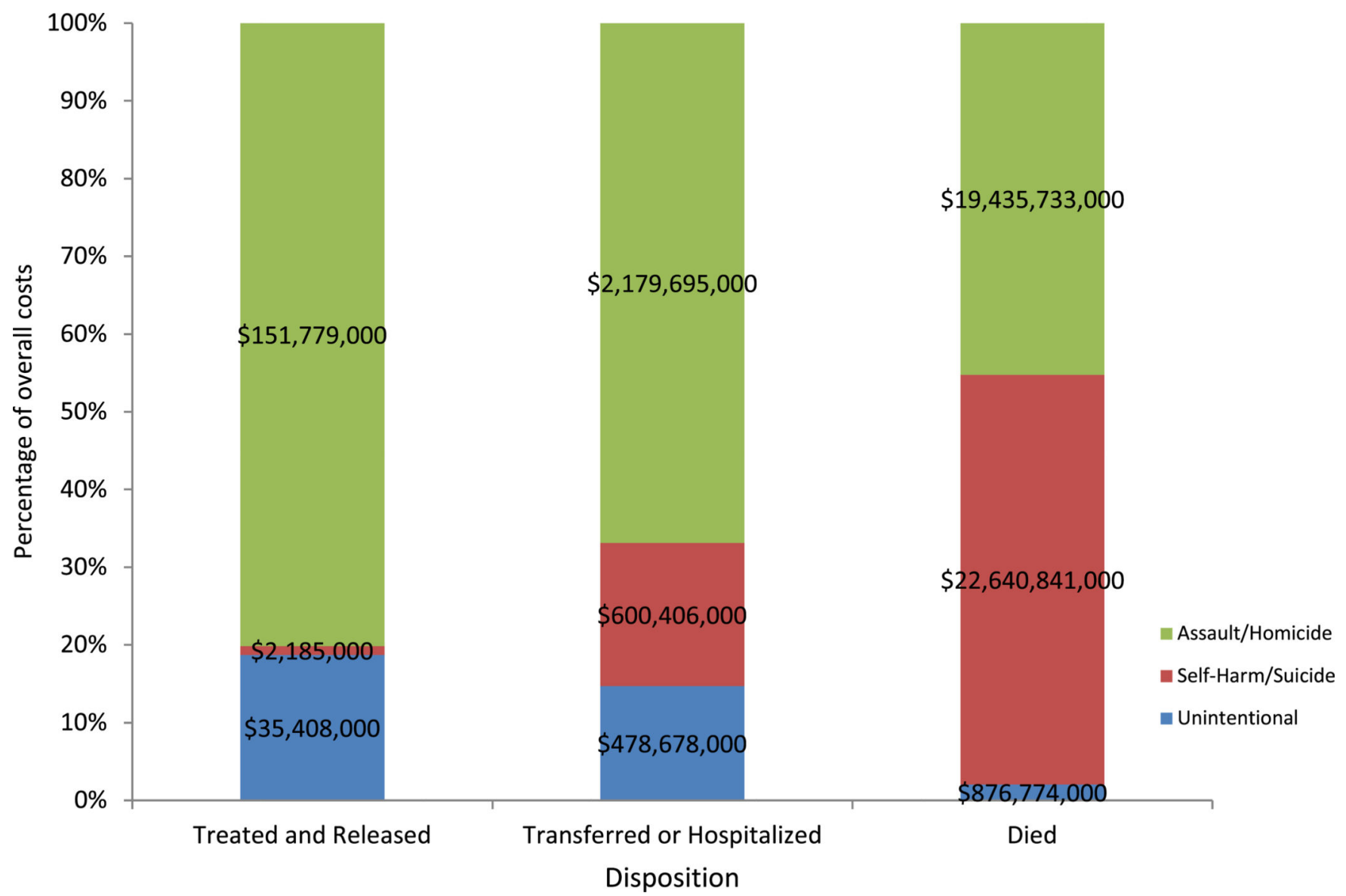

Fig. 6.

Percentage distribution of total lifetime costs, by intent of firearm injury and disposition, United States, 2010-2012. Data source: CDC WISQARS Cost of Injury reports. Overall costs of injury are derived from work loss and medical costs combined, and are based on 2010-2012 average annual firearm injuries and deaths. Note: Total lifetime costs for fatal firearm injuries sum to $\$ 44,041,023,000$; total lifetime cost for nonfatal firearm injuries sum to $\$ 4,251,361,000$. By intent, unintentional firearm injuries accounted for $\$ 1,390,860,000$ in total lifetime costs; self-harm/suicide firearm injuries accounted for $\$ 23,243,432,000$ in total lifetime costs; and assault/homicide firearm injuries accounted for $\$ 21,767,207,000$ in lifetime costs. 


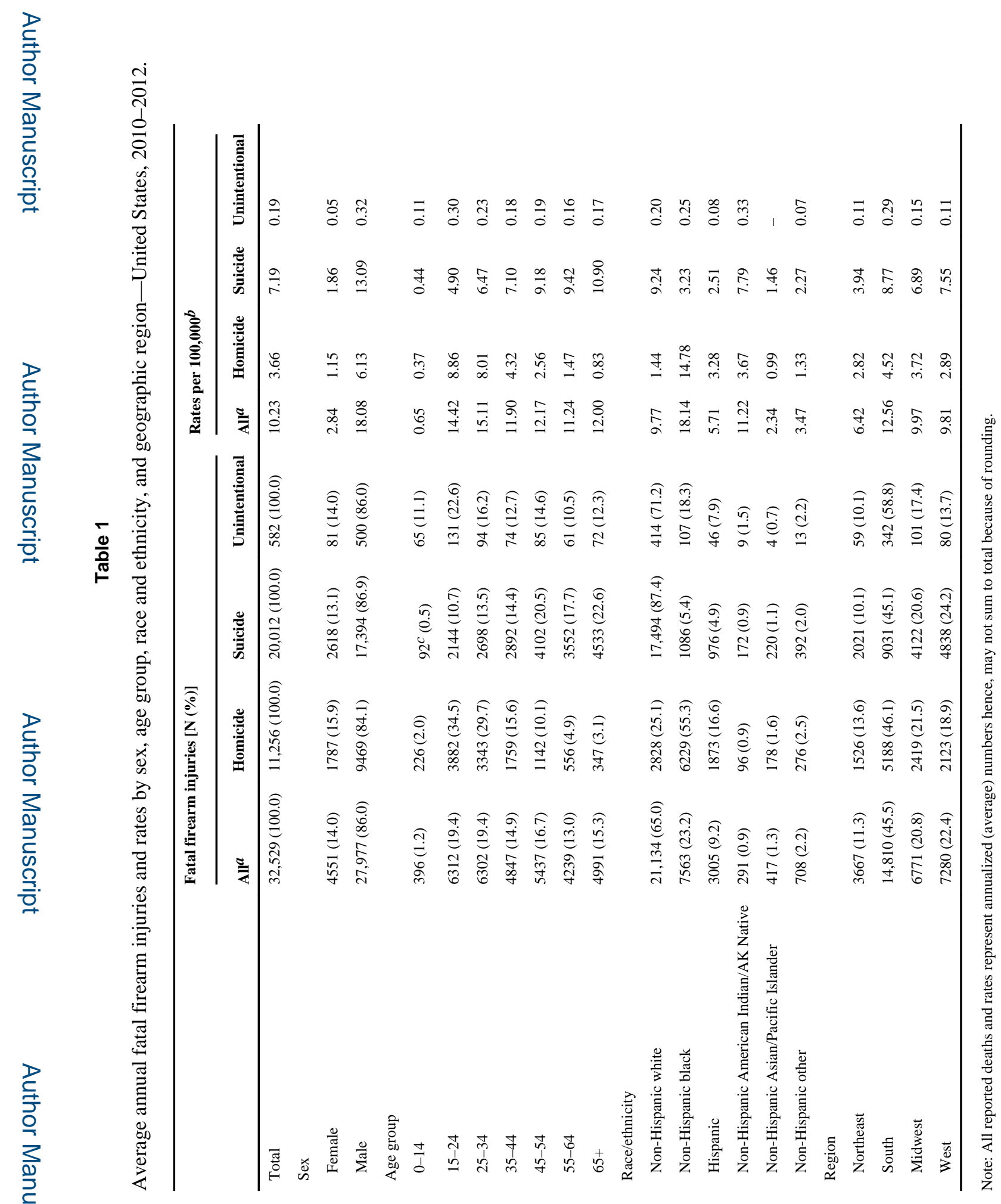

Prev Med. Author manuscript; available in PMC 2016 October 01. 


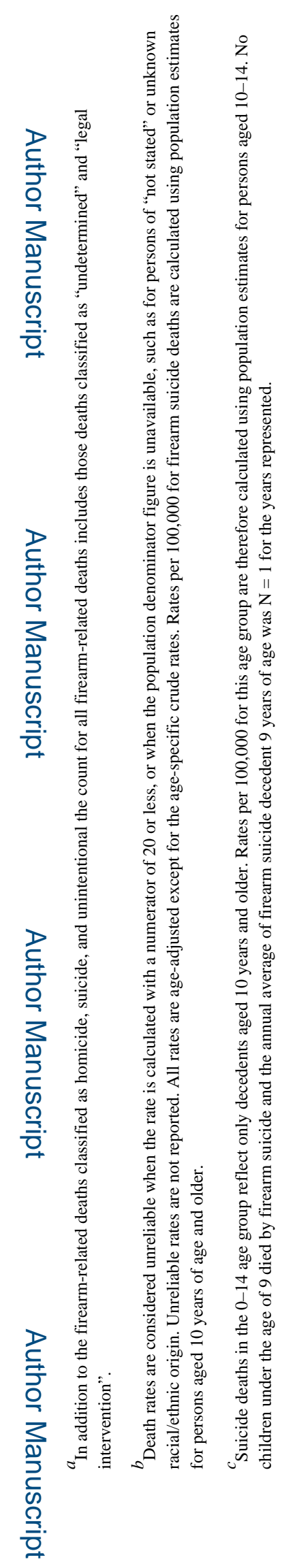

Prev Med. Author manuscript; available in PMC 2016 October 01. 


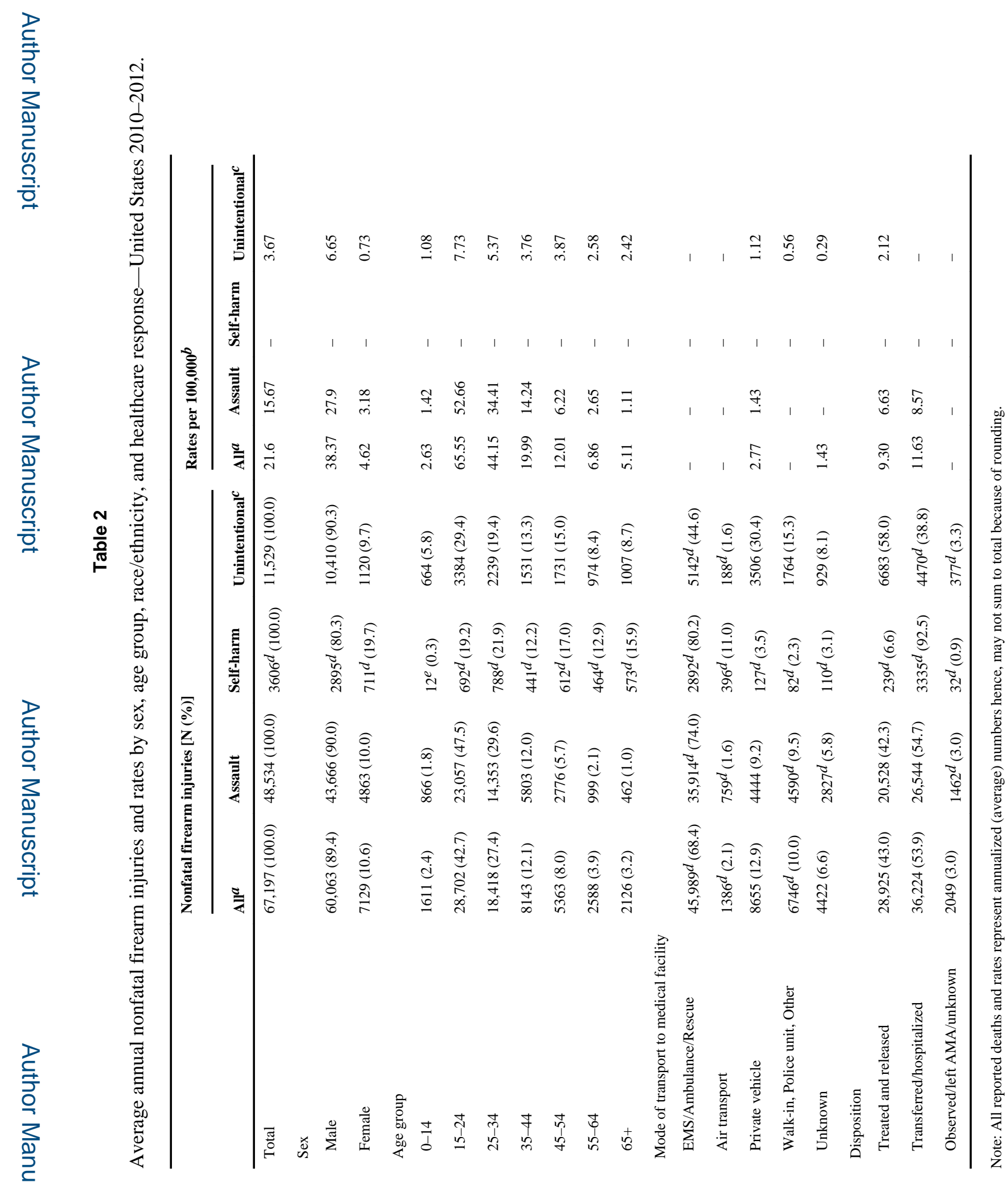

Prev Med. Author manuscript; available in PMC 2016 October 01. 


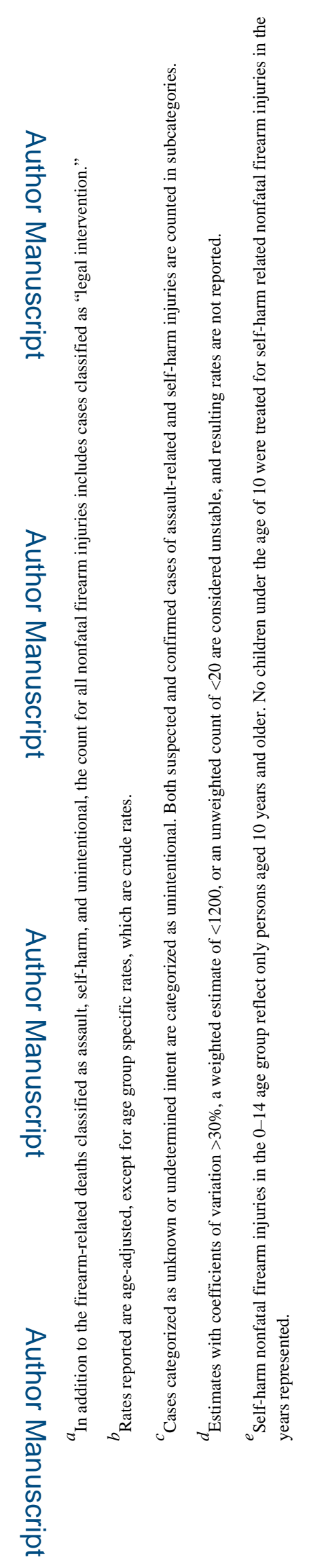

Prev Med. Author manuscript; available in PMC 2016 October 01. 


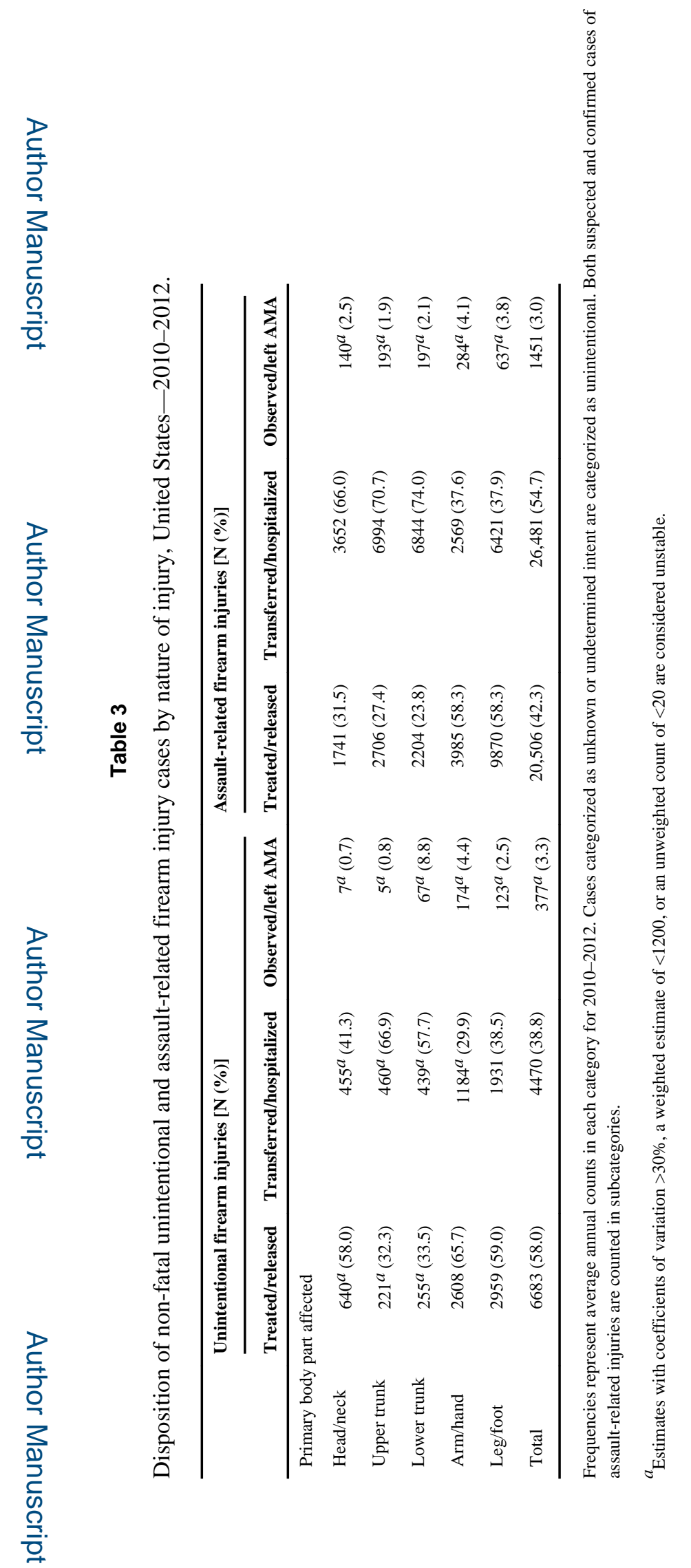

Prev Med. Author manuscript; available in PMC 2016 October 01. 